\title{
CRACK INITIATION PREDICTION FOR V-NOTCHES UNDER MIXED-MODE LOADING IN BRITTLE MATERIALS
}

\author{
JIA LI AND XIAO-BING ZHANG
}

\begin{abstract}
In this work, crack initiation from a notch tip in elements subjected to a mixed mode loading was studied. Two phenomenological criteria, based on a concept of Leguillon, were proposed and applied to predict crack initiation from a $V$-notch tip in a brittle or quasibrittle material. The first criterion, called in this paper the $G-\sigma$ criterion, makes use of the critical strain energy release rate and the ultimate tensile stress as the main parameters of fracture. It requires calculations of strain energy release rate after an infinitesimal crack kinking. By means of some numerical effort, we established the indispensable relationships between the strain energy release rate after crack kinking and the remote mixed mode loads. These relationships enabled us to apply directly the proposed criterion to mixed-mode loaded notches. We also modified the strain energy density criterion proposed by Sih on the basis of Leguillon's concept to form a criterion capable of predicting the crack initiation from a $V$-notch tip. This criterion, which we call the $S-\sigma$ criterion, benefits from its simplicity in applications. Confrontation with experimental data shows that both the criteria enable reasonably accurate prediction of fracture in elements with $V$-notches subjected to mixed mode loading. Consequently, they can be effectively applied in practical engineering applications.
\end{abstract}

\section{Introduction}

In engineering applications, the failure prediction of a structure depends not only on the strengths of the materials with which it is built, but also on the local stress concentrations [Bazant 2000]. For brittle or quasibrittle elastic materials, it is clear that the crack initiation from a stress concentration area is a critical moment in the structure service life. In the case where a $V$-shaped notch exists in a structure component, the crack initiation from the notch tip requires careful analyses because the stress field near the notch tip is a singular one and therefore this area is a potential origin of the crack initiation.

Different crack initiation criteria for $V$-notches have been reported in the literature. A fairly obvious choice consists in establishing notch fracture criteria on the basis of the critical values of the notch stress intensity factors [Reedy and Guess 1995; Dunn et al. 1997b; Dunn et al. 1997a]. Recently, attention has been focused on finite-fracture based criteria in which a finite-length crack or a finite damage zone is introduced. In general, these criteria have been drawn from the work of [McClintock 1958] on crack growth in ductile materials. The initial idea of McClintock was that in order to avoid the singularity of the near-tip fields, one can choose the normal strain $\varepsilon_{\theta \theta}$ at some small distance $l$ measured from the crack tip as a fracture parameter: the crack propagates once it reaches its critical value. Subsequently, numerous variants of this criterion were reported in the literature. For crack propagations, several criteria were proposed by choosing the normal tensile stress $\sigma_{\theta \theta}$ [Ritchie et al. 1973; Kosai et al. 1993] or the

Keywords: fracture criteria, brittle fracture, v-shaped notches, mixed mode loading, crack initiation. 
tensile stress $\sigma_{\theta \theta}$ and the shear stress $\sigma_{r \theta}$ [Mroz and Seweryn 1998], at a finite distance $l$ from the crack tip as fracture parameters. For crack onset from a notch tip, similar criteria were established (see the summary made in [Seweryn and Lukaszewicz 2002]). Apart from these criteria, the strain energy release rate at a virtually created crack tip of a finite length $l$ and the strain energy density factor $S$ have also been introduced into the fracture criteria [Palaniswamy and Knauss 1972; Seweryn and Lukaszewicz 2002]. In all these criteria, the finite length $l$ is an inevitable parameter that enables us to deal with stress singularities different from $-1 / 2$. However, the physical interpretation of this length is rather vague. It was often considered as a material parameter related to the microstructure of the material, as in the grain size in metals. In common practice, this characteristic length is often calculated by the Irwin concept [Irwin 1968] which gives rise to formulas such as $l=E G_{c} / \sigma_{c}^{2}$, with $E$ being the Young modulus, $G_{c}$ the critical value of strain energy release rate, $\sigma_{c}$ the limit failure stress.

[Leguillon 2002] pointed out that if the concept of the finite fracture mechanics is applicable to the crack initiation analysis, the finite characteristic length $l$ is not only a material-dependent constant. In fact, it depends also on the stress distribution in the structure, that is, on the structure geometry and the remote loading. In his analysis of crack onset at a $V$-notch tip, he argued that an admissible length $l$ can be determined by $l=E G_{c} /\left(\bar{K} \sigma_{c}^{2}\right)$, where $\bar{K}$ depends on the notch opening angle. This analysis enabled him to propose a crack onset criterion, in which both the tensile stress and the incremental strain energy release rate at a finite distance $l$ should reach their critical values $\sigma_{c}$ and $G_{c}$ simultaneously. This criterion is self-consistent. In fact, these conditions are sufficient to determine both the characteristic distance $l$ and the critical remote loads. This criterion provides good results in the prediction of crack onset in $V$-notched structures under pure mode $I$ loading [Leguillon 2002; Leguillon and Yosibash 2003].

In this paper, we apply the concept developed by Leguillon to the crack onset analysis for $V$-notched plates subjected to mixed-mode loading. A criterion based on the calculation of the strain energy release rate at a virtual crack tip issued from the notch tip and the tensile stress at the same point in the configuration without the virtual crack, named in this paper the $G-\sigma$ criterion, is first proposed. The main difficulty in the application of this criterion is to evaluate the strain energy release rate. Important numerical effort has been made to this end.

In order to avoid too much numerical computation, another crack onset criterion, which we designate the $S$ - $\sigma$ criterion, is here proposed on the basis of the strain energy density criterion of Sih [Sih 1974]. The main idea is to modify Sih's criterion by using the Leguillon concept in order to deal with stress singularities different from $-1 / 2$. This criterion provides a simpler technique to the prediction of crack initiation from a notch tip. Comparing with the experimental data, both criteria give satisfactory predictions for the limit loads as well as for the crack deviation angles. Finally, the efficiency of these criteria is discussed at the end of this paper.

\section{Experimental basis}

Numerous experimental studies have been published in the topic of evaluating structure failures with $V$-shaped notches [Broughton et al. 1990; Seweryn et al. 1997; Bansal and Kumosa 1998]. In this work, the experimental results obtained by [Seweryn et al. 1997] were chosen to verify the efficiency of the proposed criteria. In [Seweryn et al. 1997], symmetric plane specimens with $V$-shaped notches were made of polymethyl metacrylate (PMMA). The selection of the PMMA material was essentially due 
to its brittle characteristics, since it allows the possibility of cutting notches of a small radius (about $0.01 \mathrm{~mm}$ ). The mechanical properties of the material are as follows:

(i) Young's modulus: $E=3300 \mathrm{MPa}$;

(ii) Poisson ratio: $v=0.35$;

(iii) limit failure stress: $\sigma_{c}=102.8 \mathrm{MPa}$;

(iv) critical strain energy release rate: $G_{c}=0.384 \mathrm{MPa} \cdot \mathrm{mm}$.

The geometry of the specimens is shown in Figure 1. Imposed displacements were applied at two ends of the specimens by using a specially designed device. The mode mixity was adjusted by varying the proportion of the horizontal and vertical displacement components, that is, the ratio of shear to tensile forces.

\section{Leguillon's and modified Sih's criteria for notches under mode-I loading}

In this section, we first review the crack onset criterion proposed by [Leguillon 2002]. Then the Leguillon concept is used to modify the criterion of Sih in order to form another crack onset criterion for a $V$-shaped notch under pure tensile loading. Finally, the critical loads for crack onset predicted by using these criteria are compared with the experimental data.

Consider first a plane sharp notch in a linear elastic medium with vertex angle $2 \alpha$ and a polar coordinate system $(r, \theta)$ with the origin at the tip of the notch (Figure 2). The dominant displacements $u_{i}$ and stresses $\sigma_{i j}$ near the notch tip can be written as follows:

$$
\begin{aligned}
u_{i} & =\frac{k_{1} r^{\lambda_{1}}}{2 \mu(2 \pi)^{1-\lambda_{1}}} \tilde{u}_{1 i}(\theta)+\frac{k_{2} r^{\lambda_{2}}}{2 \mu(2 \pi)^{1-\lambda_{2}}} \tilde{u}_{2 i}(\theta) \\
\sigma_{i j} & =\frac{k_{1}}{(2 \pi r)^{1-\lambda_{1}}} \tilde{\sigma}_{1 i j}(\theta)+\frac{k_{2}}{(2 \pi r)^{1-\lambda_{2}}} \tilde{\sigma}_{2 i j}(\theta),
\end{aligned}
$$

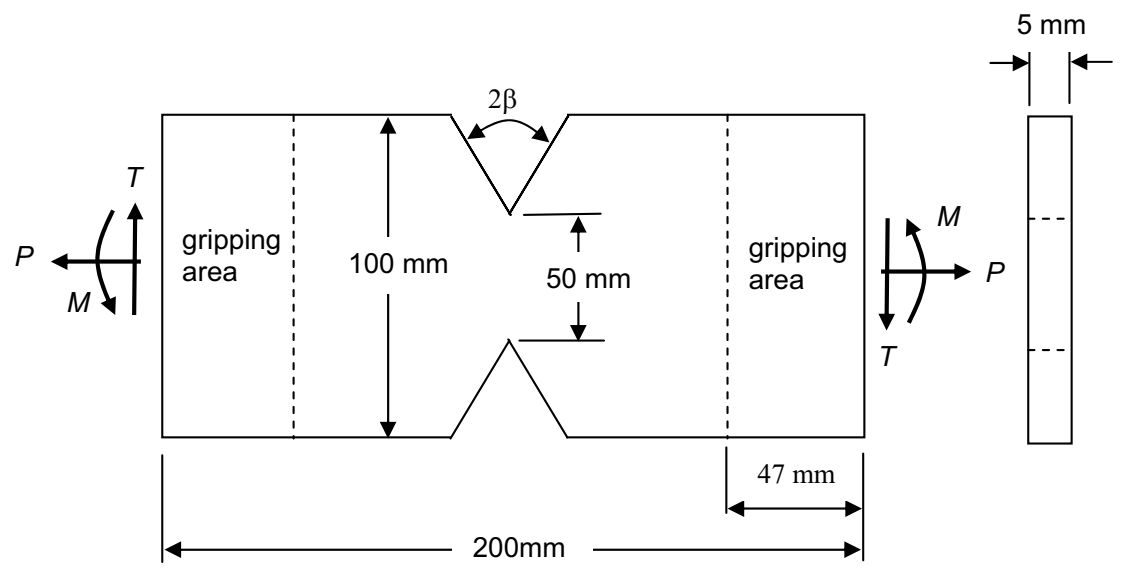

Figure 1. Geometry of specimens with $V$-notches. 


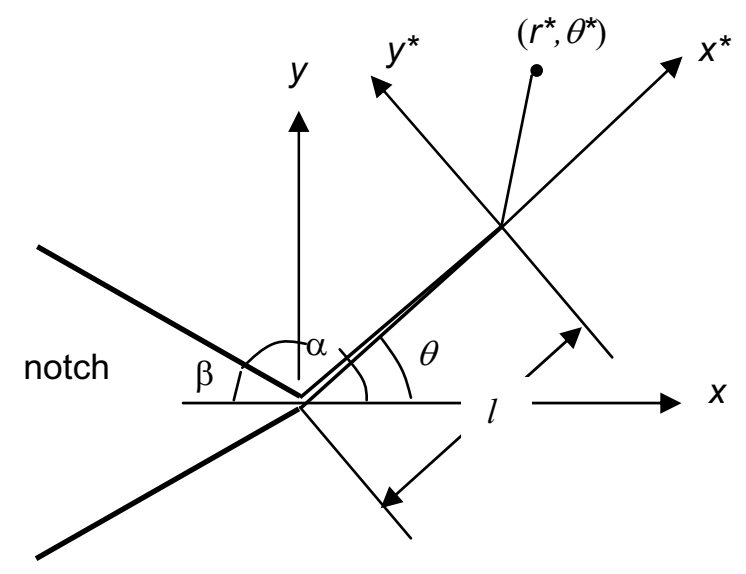

Figure 2. Notch geometry, virtual crack and coordinate systems.

where $\mu$ is the shear modulus, $\tilde{u}_{1 i}(\theta), \tilde{u}_{2 i}(\theta), \tilde{\sigma}_{1 i j}(\theta)$ and $\tilde{\sigma}_{2 i j}(\theta)$ are trigonometric functions of $\theta$. The exponents $\lambda_{1}$ and $\lambda_{2}$ describe the near-tip stress singularities and are given by the characteristic equations

$$
\begin{aligned}
& \lambda_{1} \sin 2 \alpha+\sin 2 \lambda_{1} \alpha=0, \\
& \lambda_{2} \sin 2 \alpha-\sin 2 \lambda_{2} \alpha=0 .
\end{aligned}
$$

The coefficients $k_{1}$ and $k_{2}$ are the so-called notch stress intensity factors, defined as

$$
\begin{aligned}
& k_{1}=\lim _{r \rightarrow 0}(2 \pi r)^{1-\lambda_{1}} \sigma_{\theta \theta}(r, \theta=0), \\
& k_{2}=\lim _{r \rightarrow 0}(2 \pi r)^{1-\lambda_{2}} \sigma_{r \theta}(r, \theta=0) .
\end{aligned}
$$

Among these components, we give the explicit expression of $\sigma_{\theta \theta}$ that is useful in later formulations:

$$
\begin{aligned}
\sigma_{\theta \theta}=\frac{k_{1}}{(2 \pi r)^{1-\lambda_{1}} C_{1}}(\cos (1+\lambda) \alpha \cos (1-\lambda) \theta-\cos (1-\lambda) \alpha \cos (1+\lambda) \theta) \\
\\
+\frac{k_{2}(1+\lambda)}{(2 \pi r)^{1-\lambda_{2}} C_{2}}(-\sin (1-\lambda) \alpha \sin (1+\lambda) \theta+\sin (1+\lambda) \alpha \sin (1-\lambda) \theta)
\end{aligned}
$$

with

$$
\begin{aligned}
& C_{1}=\cos \left(1+\lambda_{1}\right) \alpha-\cos \left(1-\lambda_{1}\right) \alpha, \\
& C_{2}=\left(1+\lambda_{2}\right) \sin \left(1-\lambda_{2}\right) \alpha-\left(1-\lambda_{2}\right) \sin \left(1+\lambda_{2}\right) \alpha .
\end{aligned}
$$

In general, $\lambda_{1}$ and $\lambda_{2}$ are larger than 0.5 , therefore, the singularities at a $V$-shaped notch tip are weaker than that at a crack tip. As a consequence, the criteria for crack growth, such as the strain energy release rate criterion, are not directly applicable. Moreover, the criteria usually used for nonsingular stresses such like the maximum tensile stress criterion are not appropriate. Special criteria have to be developed for this class of problems.

3.1. Leguillon's criterion. Leguillon [2002] proposed a criterion to predict the crack initiation from a notch tip. In fact, under pure tensile loading, the incremental strain energy release rate of a newly created 
crack originated from the notch tip can be developed in the form

$$
\bar{G}=-\frac{W}{l}=c k_{1}^{2} l^{2 \lambda_{1}-1}+\ldots,
$$

where $l$ is the crack length, $W$ is the potential strain energy needed to create the crack, $c$ is a constant depending on the notch-opening angle and the material parameters. Its values have been tabulated by Leguillon [2002]. The crack onset condition, according to the incremental Griffith criterion, requires that

$$
\bar{G}=c k_{1}^{2} l^{2 \lambda_{1}-1} \geq G_{c} .
$$

It is clear that $\bar{G}$ tends to zero if $l$ is infinitesimally small. Therefore, there must be a jump from 0 to $l$ when crack onset occurs. From Equation (6), the length of this jump must satisfy the condition

$$
l^{2 \lambda_{1}-1} \geq \frac{G_{c}}{c k_{1}^{2}}
$$

On the other hand, under pure tensile loading, the tensile stress at the point $(r=l, \theta=0)$ is, according to $(4)$,

$$
\sigma_{\theta \theta}=k_{1}(2 \pi l)^{\lambda_{1}-1} .
$$

It is clear that the tensile stress tends to the infinity when $l$ tends to zero. According to the maximal tensile stress criterion, we have, for small $l$,

$$
\sigma_{\theta \theta}=k_{1}(2 \pi l)^{\lambda_{1}-1} \geq \sigma_{c}
$$

which leads to

$$
(2 \pi l)^{1-\lambda_{1}} \leq \frac{k_{1}}{\sigma_{c}} .
$$

For a small load represented by $k_{1}$, the conditions (7) and (10) are incompatible. When the load increases monotonically, it reaches a critical value $k_{c}$ for which both the conditions (7) and (10) hold. This leads us to determine the increment length:

$$
l=\frac{1}{(2 \pi)^{2-2 \lambda_{1}}} \frac{G_{c}}{c \sigma_{c}^{2}} .
$$

Once the incremental length $l$ determined, the critical remote load $k_{c}$ can be calculated from either (6) or (9).

3.2. Modified Sih's criterion. Sih [1974] assumed that the crack bifurcation direction from a crack tip is determined by the minimum value of strain energy density factor $S$, and the crack propagation occurs once the strain energy density factor reaches a critical value $S_{c}$. In a polar coordinate system with the origin at the crack tip, the elastic strain energy $d U$ stored in the elementary volume $d V=r d \theta d r$ is given by the relation

$$
\frac{d U}{d V}=\frac{1}{2}\left(\sigma_{r r} \varepsilon_{r r}+\sigma_{\theta \theta} \varepsilon_{\theta \theta}+2 \sigma_{r \theta} \varepsilon_{r \theta}\right)
$$

The factor $S$ is defined as

$$
S=\frac{d U}{d V} r
$$


The critical value of $S$ can be related to the critical strain energy release rate $G_{c}$ [Sih 1974], namely

$$
S_{c}=\frac{\kappa-1}{\kappa+1} \frac{1}{\pi} G_{c}, 0
$$

where

$$
\begin{cases}\kappa=(3-v) /(1+v) & \text { for plane stress } \\ 3-4 v & \text { for plane strain. }\end{cases}
$$

Therefore Sih's criterion for crack propagation can be written as follows:

- the crack bifurcation direction $\varphi$ is determined by

$$
\left.\frac{\partial S}{\partial \theta}\right|_{\theta=\varphi}=0,\left.\quad \frac{\partial^{2} S}{\partial \theta^{2}}\right|_{\theta=\varphi}>0 ;
$$

- the crack propagates once

$$
S \geq S_{c}
$$

Consider now a $V$-shaped notch under pure tensile loading. According to Equation (13), the strain energy density factor takes the form

$$
S=k_{1}^{2}(2 \pi r)^{2 \lambda_{1}-1} \tilde{S}(\theta)
$$

where $\tilde{S}(\theta)$ is a dimensionless angular function that can easily be determined by means of Equations (1) and (12). In the case of pure tensile loading, the crack initiation angle from the notch tip is $\varphi=0$. In such a case, $\tilde{S}(0)$ becomes a constant $K$ depending on the notch-opening angle. For plane stress, for example, we can write

$$
\begin{aligned}
K & =\frac{1-2 \nu \sigma+\sigma^{2}}{4 \pi E} \\
\sigma & =\frac{\left(3-\lambda_{1}\right) \cos \left(1+\lambda_{1}\right) \alpha+\left(1+\lambda_{1}\right) \cos \left(1-\lambda_{1}\right) \alpha}{\left(1+\lambda_{1}\right)\left(\cos \left(1+\lambda_{1}\right) \alpha-\cos \left(1-\lambda_{1}\right) \alpha\right)} .
\end{aligned}
$$

It is clear that $\lim _{r \rightarrow 0} S(r, 0)=0$. In order to be able to use Sih's criterion, we adopt a nonlocal length $r=l$, at this distance the crack onset condition (16) becomes

$$
S(l)=K k_{1}^{2}(2 \pi l)^{2 \lambda_{1}-1} \geq S_{c} .
$$

Therefore

$$
(2 \pi l)^{2 \lambda_{1}-1} \geq \frac{S_{c}}{K k_{1}^{2}} .
$$

Consider now the tensile stress at the ligament. According to the maximum tensile stress criterion, Equation (9) must hold.

Similarly to Leguillon's criterion, both Equations (20) and (9) must hold for a crack onset. Therefore we can obtain the crack onset length $l$ as

$$
l=\frac{1}{2 \pi} \frac{S_{c}}{K \sigma_{c}^{2}}
$$

and the critical remote load, represented by $k_{1}$, can easily be determined from either (19) or (9). 


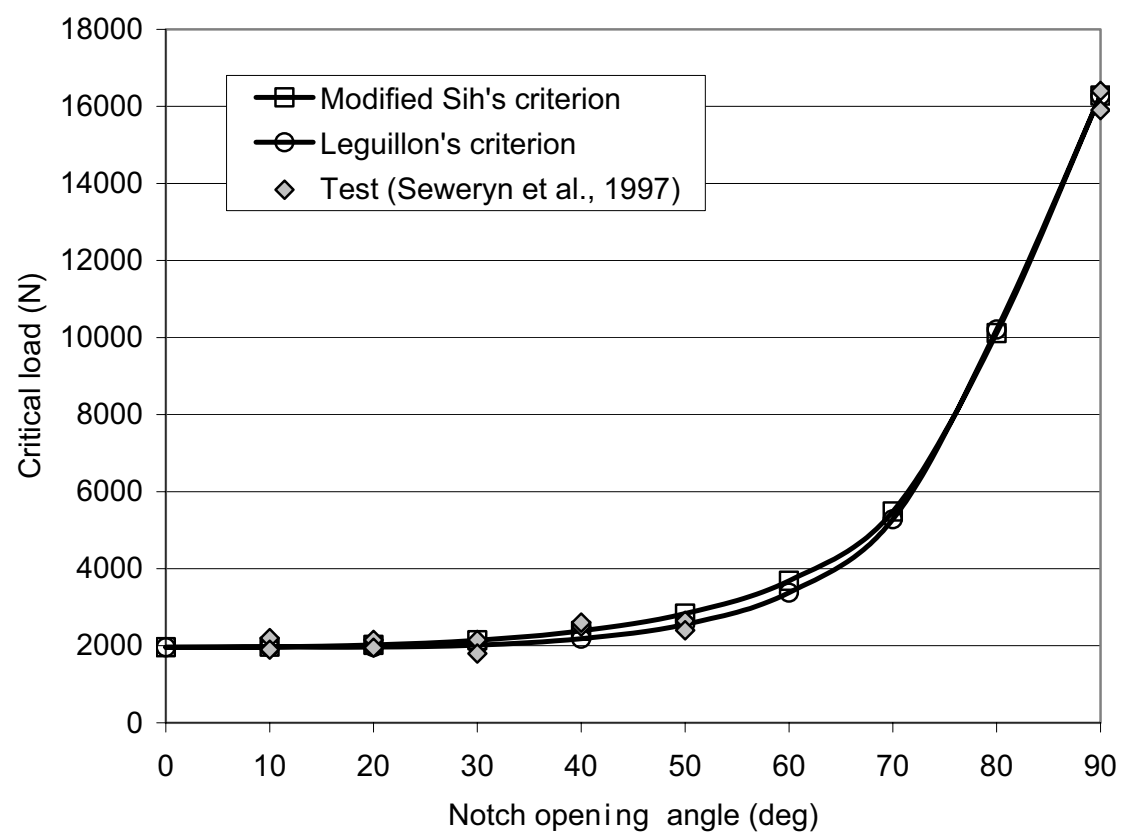

Figure 3. Failure load versus notch opening angle for $V$-notched plates under pure tensile loading

Figure 3 illustrates the comparison between the critical loads at fracture of the specimens recorded in the experimentation [Seweryn et al. 1997] and those predicted by using respectively Leguillon's criterion and modified Sih's criterion. It is shown that both criteria provide good agreement with respect to the experimental data.

\section{The $G-\sigma$ criterion for mixed-mode loaded notches}

The concept developed by Leguillon is physically reasonable and self-consistent. The main difficulty remains the evaluation of the incremental strain energy release rate $\bar{G}$. Consider the $V$-notch illustrated in Figure 2 with a crack of length $l$ issued from the notch tip in an arbitrary direction $\theta$. The incremental strain energy release rate $\bar{G}$ during the creation of this crack is the average work done by the stresses in front of the crack on the displacements of the crack edge:

$$
\bar{G}=-\frac{1}{l} \int_{0}^{l}\left(\sigma_{\theta \theta}(r, \theta) u_{\theta}^{*}(l-r, \pi)+\sigma_{r \theta}(r, \theta) u_{r}^{*}(l-r, \pi)\right) d r .
$$

However, the evaluation of $\bar{G}$ is associated with considerable computational difficulties. A basic problem is to determine displacements along the edges of an assumed crack. In the original work of Leguillon [2002], the evaluation of $\bar{G}$ is relied on the possibility of its expansion in an asymptotic series involving the crack length $l$. In the present work, we will not attempt to perform such an asymptotic analysis, and a different method is adopted.

Let us consider the same $V$-shaped notch. We assume that under mixed mode loading, there exists a small crack issued from the notch tip and characterized by its length $l$ and its orientation $\theta$. The 
assumption of this finite-length crack can be justified as follows [Seweryn and Lukaszewicz 2002]: due to large stresses, intensive microcracking occurs near the notch tip. This microcrack network can be replaced by an equivalent crack in order to produce a mathematically manageable model. The size of this equivalent crack is essentially within a mesoscopic scale (about several tens of microns for the used PMMA). In brittle materials, the instable propagation of this equivalent crack leads to a macroscopic crack onset.

The energy release rate of this virtual crack is defined by

$$
G=-\lim \frac{1}{\Delta l} \int_{0}^{\Delta l}\left(\sigma_{\theta \theta}^{*}(0) u_{\theta}^{*}(\pi)+\sigma_{r \theta}^{*}(0) u_{r}^{*}(\pi)\right) d r,
$$

where $\left(r^{*}, \theta^{*}\right)$ are the polar coordinates with an origin at the crack tip and $\sigma_{\theta \theta}^{*}, \sigma_{r \theta}^{*}, u_{\theta}^{*}$ and $u_{r}^{*}$ are the stress and displacement components in the above coordinates. Taking into consideration only the first terms of the asymptotic expansion of the stress and displacement fields near the crack tip, one obtains

$$
G=\frac{K_{I}^{2}+K_{I I}^{2}}{E^{\prime}}
$$

where $K_{I}$ and $K_{I I}$ are the stress intensity factors at the newly created crack tip, with

$$
E^{\prime}= \begin{cases}E & \text { for plane stress } \\ \frac{E}{1-v^{2}} & \text { for plane strain. }\end{cases}
$$

A criterion of a macroscopic crack initiation can therefore be established on the basis of the energy release rate at the tip of the virtual crack. By adopting the Leguillon's concept, this criterion can be described as follows:

Crack onset occurs from the notch tip if at a neighboring point $(\varphi, l)$,

(i) the circumferential tensile stress reaches the material critical strength $\sigma_{c}$;

(ii) the strain energy release rate of a virtual crack, emanated from the notch tip to this point, reaches the critical toughness $G_{c}$;

(iii) the strain energy release rate reaches a maximal value among all possible crack onset directions.

Therefore, the application of this criterion consists in evaluating the crack initiation angle $\varphi$, the crack onset length $l$ and the critical remote load $F$ from the relationships

$$
\begin{aligned}
\sigma_{\theta \theta}(F, l, \varphi) & =\sigma_{c} \\
G(F, l, \varphi) & =G_{c} \\
\frac{\partial G(F, l, \varphi)}{\partial \varphi} & =0, \quad \frac{\partial G^{2}(F, l, \varphi)}{\partial \varphi^{2}}<0 .
\end{aligned}
$$

This criterion is called hereafter the $G-\sigma$ criterion.

The key factor in applying this criterion is to evaluate the strain energy release rate for the virtual crack emanating from the $V$-notch tip. To this end, we write, from a dimensional analysis, the relationships 
between the stress intensity factors $\left(k_{1}, k_{2}\right)$ at the $V$-notch tip and $\left(K_{I}, K_{I I}\right)$, those at the virtual crack tip, for infinitesimal $l$ :

$$
\begin{gathered}
K_{I}=b_{11} k_{1} l^{\lambda_{1}-\frac{1}{2}}+b_{12} k_{2} l^{\lambda_{2}-\frac{1}{2}}, \\
K_{I I}=b_{21} k_{1} l^{\lambda_{1}-\frac{1}{2}}+b_{22} k_{2} l^{\lambda_{2}-\frac{1}{2}},
\end{gathered}
$$

where $b_{i j}$ are dimensionless functions depending on the crack deviation angle $\varphi$.

From Equations (24) and (27) we obtain

$$
E^{\prime} G=c_{1}(\varphi) k_{1}^{2} l^{2 \lambda_{1}-1}+c_{2}(\varphi) k_{1} k_{2} l^{\lambda_{1}+\lambda_{2}-1}+c_{3}(\varphi) k_{2}^{2} l^{2 \lambda_{2}-1},
$$

where $c_{1}(\varphi), c_{2}(\varphi)$ and $c_{3}(\varphi)$ result from combination of $b_{i j}$ and therefore are $\varphi$-dependent functions. In order to determine these functions, we carried out detailed numerical calculations by establishing finite element models. The idea is to mesh carefully a notched plate with a branched crack. Then we calculate accurately the strain energy release rates at the crack tip by varying the crack length, the crack direction, and the mode mixity of the remote loads. These numerical data are correlated with Equation (28) by using a least square procedure in order to determine accurately the functions $c_{i}(\varphi)$. This approach seems to be somewhat onerous and lacking in elegance. However, with the extraordinary capacity of actual computers and finite element codes, a linear elastic calculation is quite rapid. Combining this with an automatic meshing procedure, the entire computational work is not extensively time-consuming.

In the numerical simulations, the specimens used in [Seweryn et al. 1997] were meshed as illustrated in Figure 4. The lower specimen edge was fixed while along the upper specimen edge the enforced displacements were prescribed in the direction of $y$-axis for tension and $x$-axis for shear. The tensile and shearing load components $P$ and $T$ were calculated by integrating the resulting stresses across the
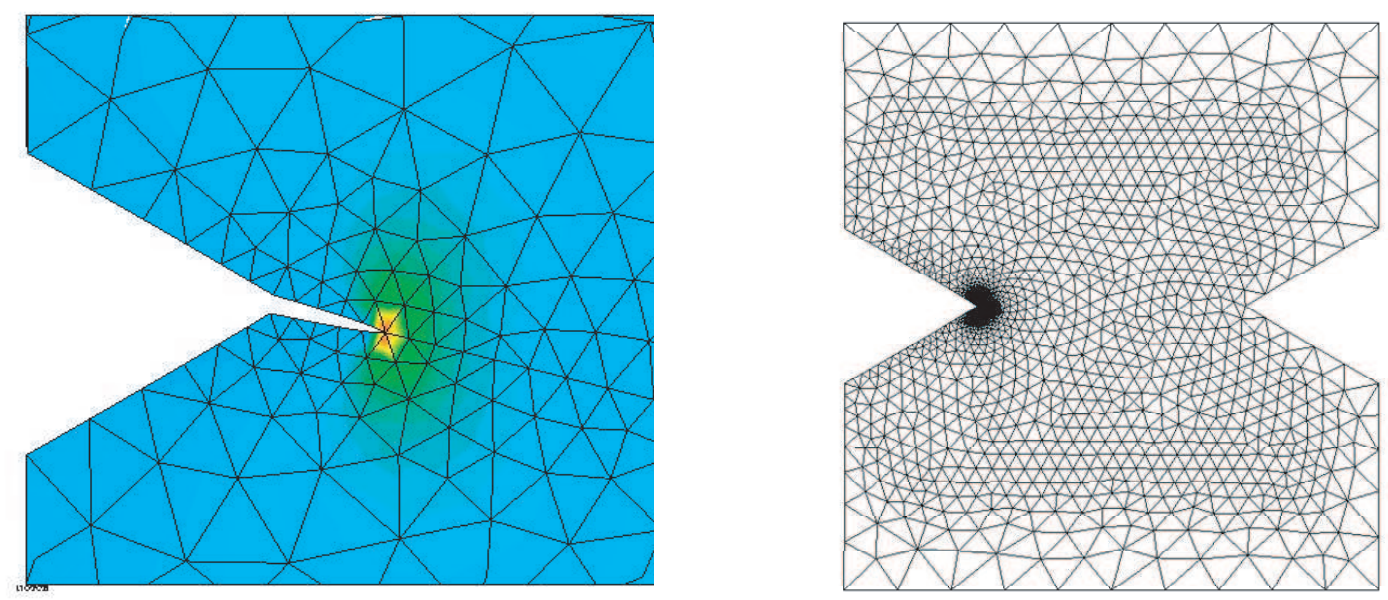

Figure 4. Typical meshing of the specimens and the zoom around the branched crack. 
specimen width. Therefore the resulting force $F$ is related to $P$ and $T$ by

$$
F=\sqrt{P^{2}+T^{2}}, \quad P=F \cos \psi, \quad T=F \sin \psi,
$$

where $\psi$ denotes the application angle of the resulting force. The notch stress intensity factors $k_{1}$ and $k_{2}$ are related to the remote loading by

$$
\begin{aligned}
& k_{1}=\xi_{1} P=\xi_{1} F \cos \psi \\
& k_{2}=\xi_{2} T=\xi_{2} F \sin \psi,
\end{aligned}
$$

where the coefficients $\xi_{1}$ and $\xi_{2}$ for these specimens have been computed and tabulated by [Seweryn and Lukaszewicz 2002].

A highly accurate method, the so-called $G-\Theta$ method [Destuynder et al. 1983; Suo and Combescure 1992] and implemented in the finite element code CAST3M (software edited by the Commissariat d'Énergie Atomique of France), was used to calculate the strain energy release rates for branched cracks. To be precise, 30 values of $G$ were calculated for each crack initiation angle with 5 crack lengths, varying from $0.05 \mathrm{~mm}$ to $0.5 \mathrm{~mm}$, and 6 load mixities, varying from pure tension to pure shear. These values of $G$ were then input into a least square program to obtain $c_{i}(\varphi)$. An example of the fitting procedure is given in Figure 5 that shows the correlation between the numerical values of $G$ and Equation (28) for a $60^{\circ}$ notch under pure mode I loading. In this Figure, the circles represent the FE results, and the line represents the fitting curve according to (28). We can see that the fitting is highly accurate. This accuracy can be obtained for nearly all the cases we treated. Consequently, we can confirm that the present numerical correlation method is suitable.

The numerical values of $c_{i}(\varphi)$ are plotted in Figures 6 and 7. For reasons of symmetry, i.e. $c_{1}(\varphi)=$ $c_{1}(-\varphi), c_{2}(\varphi)=-c_{2}(-\varphi), c_{3}(\varphi)=c_{3}(-\varphi)$, only $c_{i}(\varphi)$ in the interval $[-\alpha, 0]$ are presented. From these

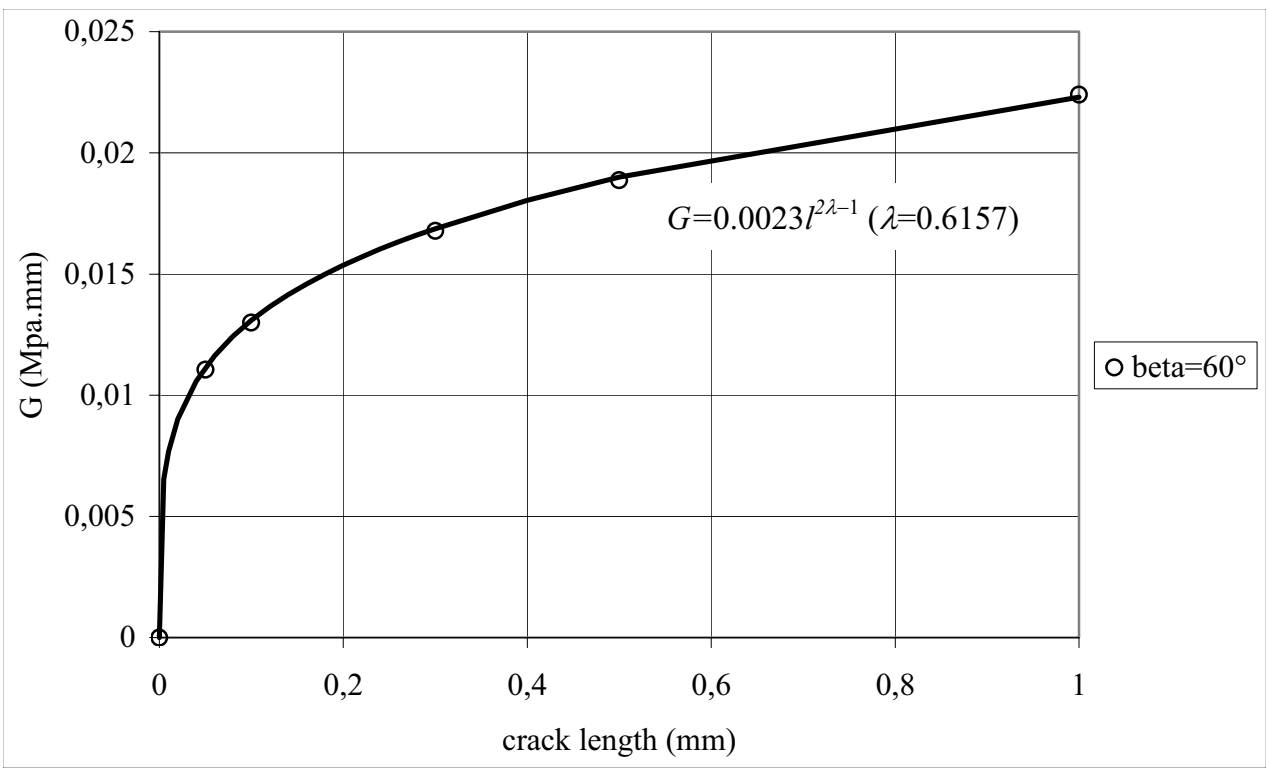

Figure 5. Correlation between the numerical values of $G$ and Equation (28). 

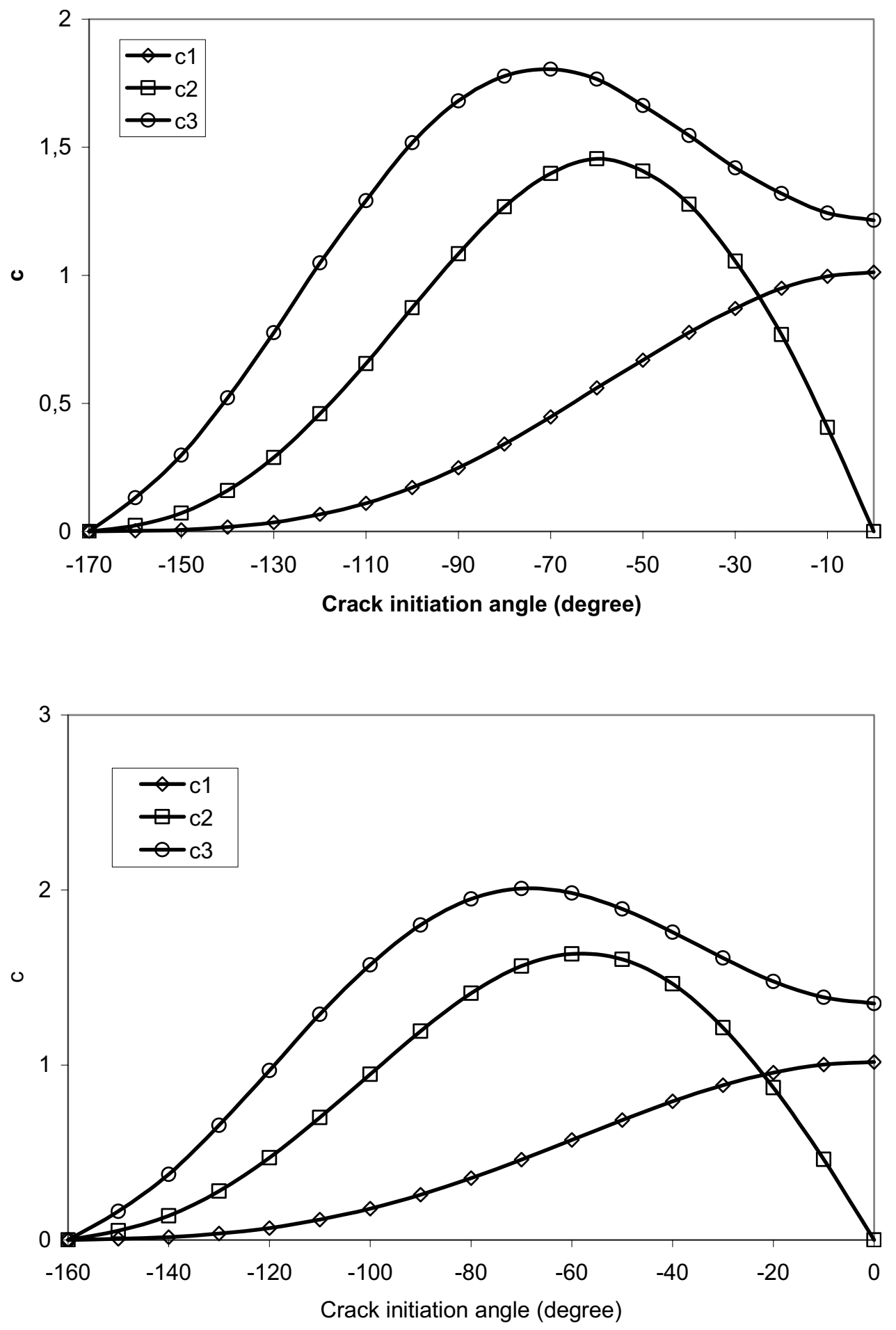

Figure 6. Variation of $c_{i}$ as function of crack initiation angle: (top) $\beta=10^{\circ}$, (bottom) $\beta=20^{\circ}$. 

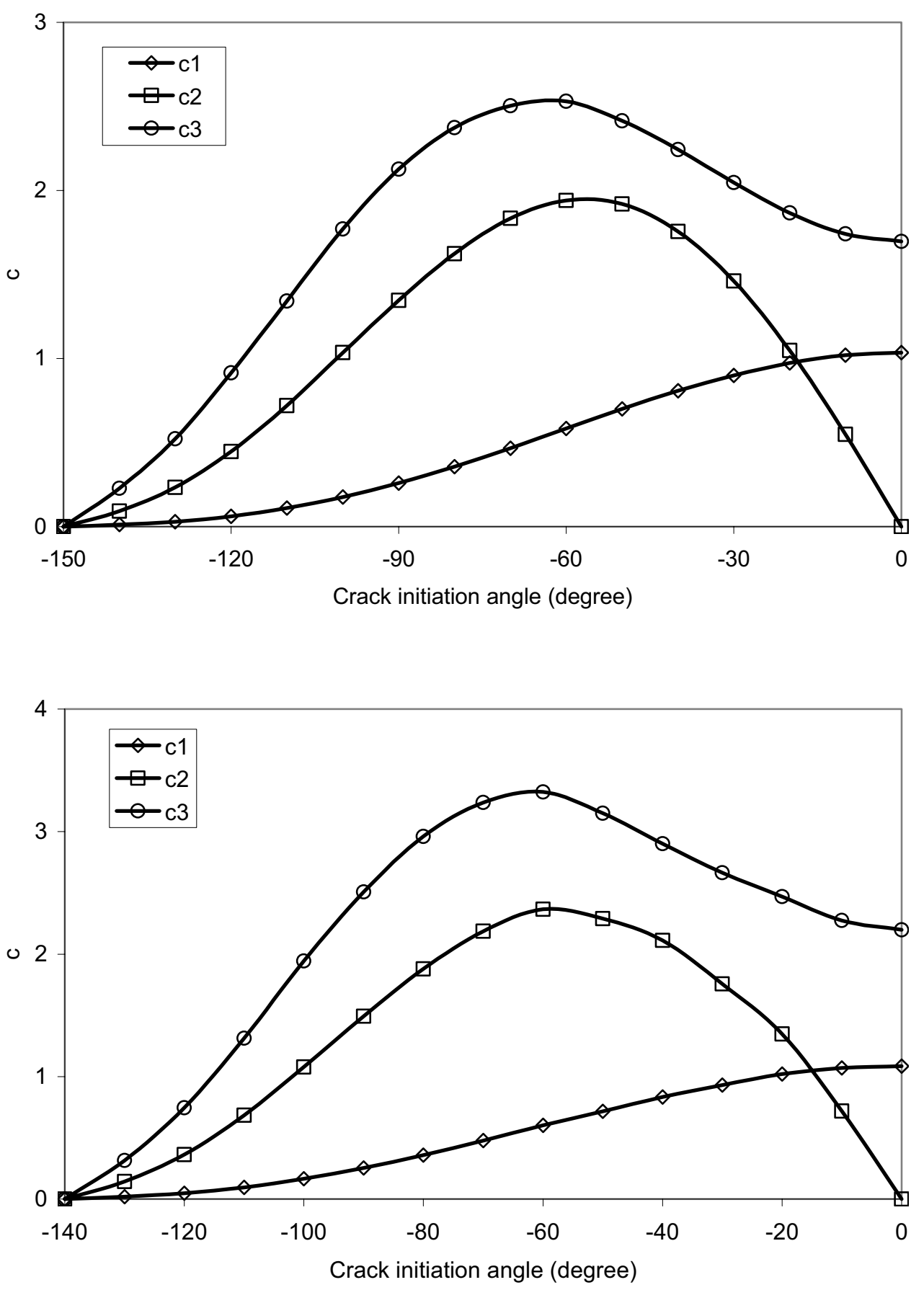

Figure 7. Variation of $c_{i}$ as function of crack initiation angle: (top) $\beta=30^{\circ}$; (bottom) $\beta=40^{\circ}$. 
results, the strain energy release rate for any branched crack from the notch tip of a small length can easily be calculated from Equation (28).

By introducing Equations (4), (28) and (29) into Equation (26), we rewrite explicitly the conditions in the $G-\sigma$ criterion:

$$
\begin{array}{r}
\frac{\xi_{1} \cdot F \cos \psi}{(2 \pi l)^{1-\lambda_{1}} C_{1}}(\cos (1+\lambda) \alpha \cos (1-\lambda) \theta+\cos (1-\lambda) \alpha \cos (1+\lambda) \theta) \\
+\frac{\xi_{2} \cdot F \sin \psi(1+\lambda)}{(2 \pi l)^{1-\lambda_{2}} C_{2}}(-\sin (1-\lambda) \alpha \sin (1+\lambda) \theta+\sin (1+\lambda) \alpha \sin (1-\lambda) \theta)=\sigma_{c} \\
F^{2}\left(\left(\xi_{1} \cos \psi\right)^{2} c_{1}(\varphi) l^{2 \lambda_{1}-1}+\xi_{1} \xi_{2} \cos \psi \sin \psi c_{2}(\varphi) l^{\lambda_{1}+\lambda_{2}-1}+\left(\xi_{2} \sin \psi\right)^{2} c_{3}(\varphi) l^{2 \lambda_{2}-1}\right)=G_{c} \\
\left(\xi_{1} \cos \psi\right)^{2} c_{1}^{\prime}(\varphi) l^{2 \lambda_{1}-1}+\xi_{1} \xi_{2} \cos \psi \sin \psi c_{2}^{\prime}(\varphi) l^{\lambda_{1}+\lambda_{2}-1}+\left(\xi_{2} \sin \psi\right)^{2} c_{3}^{\prime}(\varphi) l^{2 \lambda_{2}-1}=0 \\
\left(\xi_{1} \cos \psi\right)^{2} c_{1}^{\prime \prime}(\varphi) l^{2 \lambda_{1}-1}+\xi_{1} \xi_{2} \cos \psi \sin \psi c_{2}^{\prime \prime}(\varphi) l^{\lambda_{1}+\lambda_{2}-1}+\left(\xi_{2} \sin \psi\right)^{2} c_{3}^{\prime \prime}(\varphi) l^{2 \lambda_{2}-1}<0 .
\end{array}
$$

These transcendent equations can be resolved by using numerical methods such like the NewtonRaphson method.

\section{The $S$ - $\sigma$ criterion for mixed-mode loaded notches}

The modified Sih criterion is attractive by its simplicity in applications. Under mixed mode loading, this criterion, named the $S$ - $\sigma$ criterion hereafter, can be described as follows: crack onset occurs from the notch tip if at a neighbouring point $(\varphi, l)$,

(i) the circumferential tensile stress reaches the material critical strength $\sigma_{c}$;

(ii) the strain energy density factor reaches the critical value $S_{c}$;

(iii) the strain energy density factor reaches a minimal value among all possible crack onset directions.

In plane stress, the strain energy density factor $S$ is given by

$$
\begin{aligned}
S & =\frac{1}{2}\left(\sigma_{r r} \varepsilon_{r r}+\sigma_{\theta \theta} \varepsilon_{\theta \theta}+2 \sigma_{r \theta} \varepsilon_{r \theta}\right) r \\
& =\frac{1}{2 E}\left(\sigma_{r r}^{2}+\sigma_{\theta \theta}^{2}-2 v \sigma_{r r} \sigma_{\theta \theta}+(1+\nu) \sigma_{r \theta}^{2}\right) r .
\end{aligned}
$$

At a characteristic distance $l$ from the notch tip, the strain energy density can be written as follows:

$$
S(l, \theta)=d_{1}(\theta) k_{1}^{2} l^{2 \lambda_{1}-1}+d_{2}(\theta) k_{1} k_{2} l^{\lambda_{1}+\lambda_{2}-1}+d_{3}(\theta) k_{2}^{2} l^{2 \lambda_{2}-1},
$$

where the angular functions $d_{1}(\theta), d_{2}(\theta)$, and $d_{3}(\theta)$ can readily be calculated from Equation (1). The direction of crack propagation angle $\varphi$ is defined by

$$
\begin{aligned}
& \left.\frac{\partial S}{\partial \theta}\right|_{\theta=\varphi}=\left(d_{1}^{\prime}(\theta) k_{1}^{2} l^{2 \lambda_{1}-1}+d_{2}^{\prime}(\theta) k_{1} k_{2} l^{\lambda_{1}+\lambda_{2}-1}+d_{3}^{\prime}(\theta) k_{2}^{2} l^{2 \lambda_{2}-1}\right)_{\theta=\varphi}=0 \\
& \left.\frac{\partial^{2} S}{\partial \theta^{2}}\right|_{\theta=\varphi}=\left(d_{1}^{\prime \prime}(\theta) k_{1}^{2} l^{2 \lambda_{1}-1}+d_{2}^{\prime \prime}(\theta) k_{1} k_{2} l^{\lambda_{1}+\lambda_{2}-1}+d_{3}^{\prime \prime}(\theta) k_{2}^{2} l^{2 \lambda_{2}-1}\right)_{\theta=\varphi}>0 .
\end{aligned}
$$


Consequently, the $S$ - $\sigma$ criterion is explicitly written as follows:

Find $(\varphi, l, F)$ satisfying

$$
\begin{aligned}
& \frac{\xi_{1} \cdot F \cos \psi}{(2 \pi l)^{1-\lambda_{1}} C_{1}}(\cos (1+\lambda) \alpha \cos (1-\lambda) \theta+\cos (1-\lambda) \alpha \cos (1+\lambda) \theta) \\
& +\frac{\xi_{2} \cdot F \sin \psi(1+\lambda)}{(2 \pi l)^{1-\lambda_{2}} C_{2}}(-\sin (1-\lambda) \alpha \sin (1+\lambda) \theta+\sin (1+\lambda) \alpha \sin (1-\lambda) \theta)=\sigma_{c} \\
& F^{2}\left(\left(\xi_{1} \cos \psi\right)^{2} d_{1}(\varphi) l^{2 \lambda_{1}-1}+\xi_{1} \xi_{2} \cos \psi \sin \psi d_{2}(\varphi) l^{\lambda_{1}+\lambda_{2}-1}+\left(\xi_{2} \sin \psi\right)^{2} d_{3}(\varphi) l^{2 \lambda_{2}-1}\right)=S_{c} \\
& \left(\xi_{1} \cos \psi\right)^{2} d_{1}^{\prime}(\varphi) l^{2 \lambda_{1}-1}+\xi_{1} \xi_{2} \cos \psi \sin \psi d_{2}^{\prime}(\varphi) l^{\lambda_{1}+\lambda_{2}-1}+\left(\xi_{2} \sin \psi\right)^{2} d_{3}^{\prime}(\varphi) l^{2 \lambda_{2}-1}=0 \\
& \left(\xi_{1} \cos \psi\right)^{2} d_{1}^{\prime \prime}(\varphi) l^{2 \lambda_{1}-1}+\xi_{1} \xi_{2} \cos \psi \sin \psi d_{2}^{\prime \prime}(\varphi) l^{\lambda_{1}+\lambda_{2}-1}+\left(\xi_{2} \sin \psi\right)^{2} d_{3}^{\prime \prime}(\varphi) l^{2 \lambda_{2}-1}>0
\end{aligned}
$$

We solved these nonlinear equations by using the Newton-Raphson method.

\section{Results and discussions}

The results obtained for the prediction of the failure loads of $V$-notched plates subjected to mixed loading by using the above-mentioned criteria were compared with the experimental data in [Seweryn et al. 1997]. Since the material used, the PMMA, is of brittle character, initiation of a macrocrack often leads to global failure of the element. As a consequence, a critical load to initiate a crack was considered as the fracture load of the plate.

Figures 8 and 9 presents the variation of the direction of crack initiation angles $\varphi$ versus the load application angle $\psi$ for different values of the notch-opening angle $\beta$. Experimental data were marked by points and the predicted results were presented by continue lines with points. From these figures we can observe that the predicted crack initiation directions by both the criteria agree well with the experimental results for small opening angles $\beta \leq 30^{\circ}$. However, the predicted results are less accurate for specimens with a notch opening angle $\beta=40^{\circ}$.

In Figures 10 and 11, we plot the ratios of the critical mixed mode loads $F_{c}$ to the critical tensile load $P_{c}$ determined from both the criteria as function of load application angle $\psi$. Experimental results are also represented for comparison. These figures show that both the criteria provide us with good prediction if the notch opening angle $\beta>10^{\circ}$. For $\beta=10^{\circ}$, the $S$ - $\sigma$ criterion still agrees quite well with the test data, while the $G-\sigma$ criterion gives somewhat conservative predictions when the shear loads dominate.

A common advantage of both the proposed criteria is that they are all self-consistent. No additional conditions are required in their application. This differs from the early-proposed local criteria such as the RKR criterion [Ritchie et al. 1973] or McClintock's criterion [McClintock 1958] in which a virtual process distance from the crack tip was introduced but can't be determined by the criteria themselves. This distance, however, is clearly defined with the criteria used in the present work. In general, it depends not only upon the material mechanical properties (strength and toughness), but also upon the structure configuration (geometry, loading). In the present notch cases, this characteristic length varies from $0.02 \mathrm{~mm}$ to $0.035 \mathrm{~mm}$. 

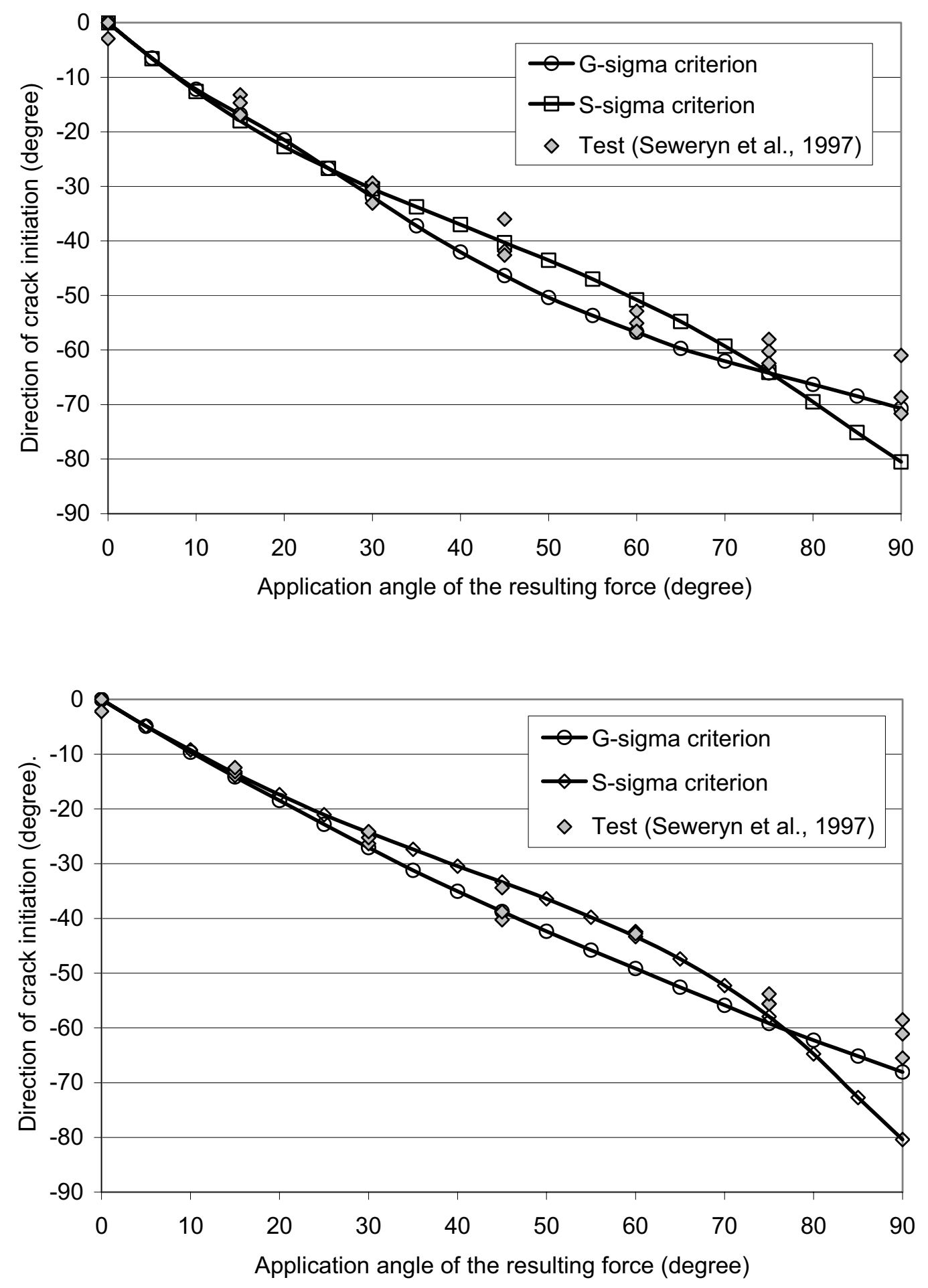

Figure 8. Direction of crack initiation versus application angle of resulting force: (top) $\beta=10^{\circ}$; (bottom) $\beta=20^{\circ}$. 

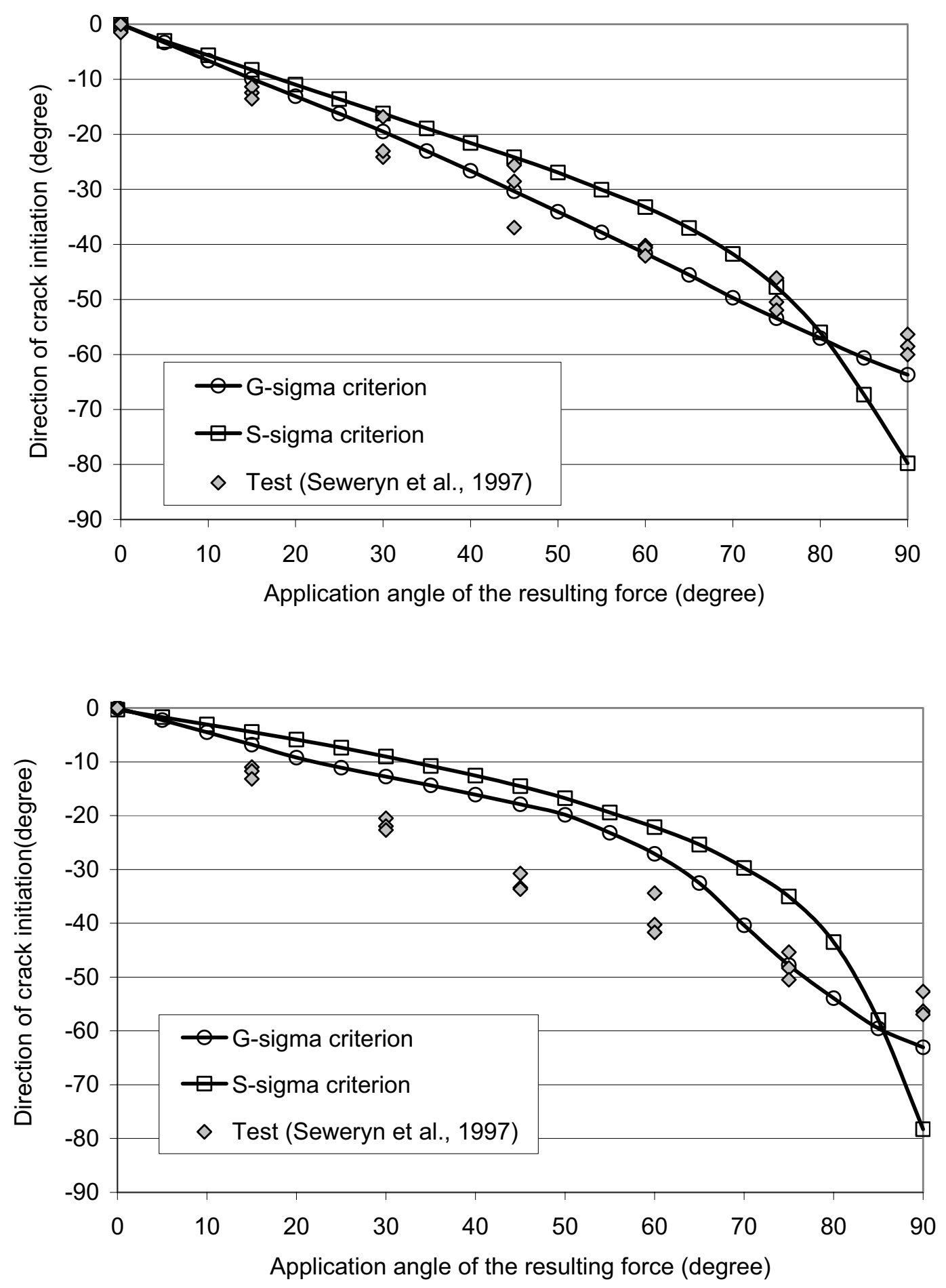

Figure 9. Direction of crack initiation versus application angle of resulting force: (top) $\beta=30^{\circ}$; (bottom) $\beta=40^{\circ}$. 

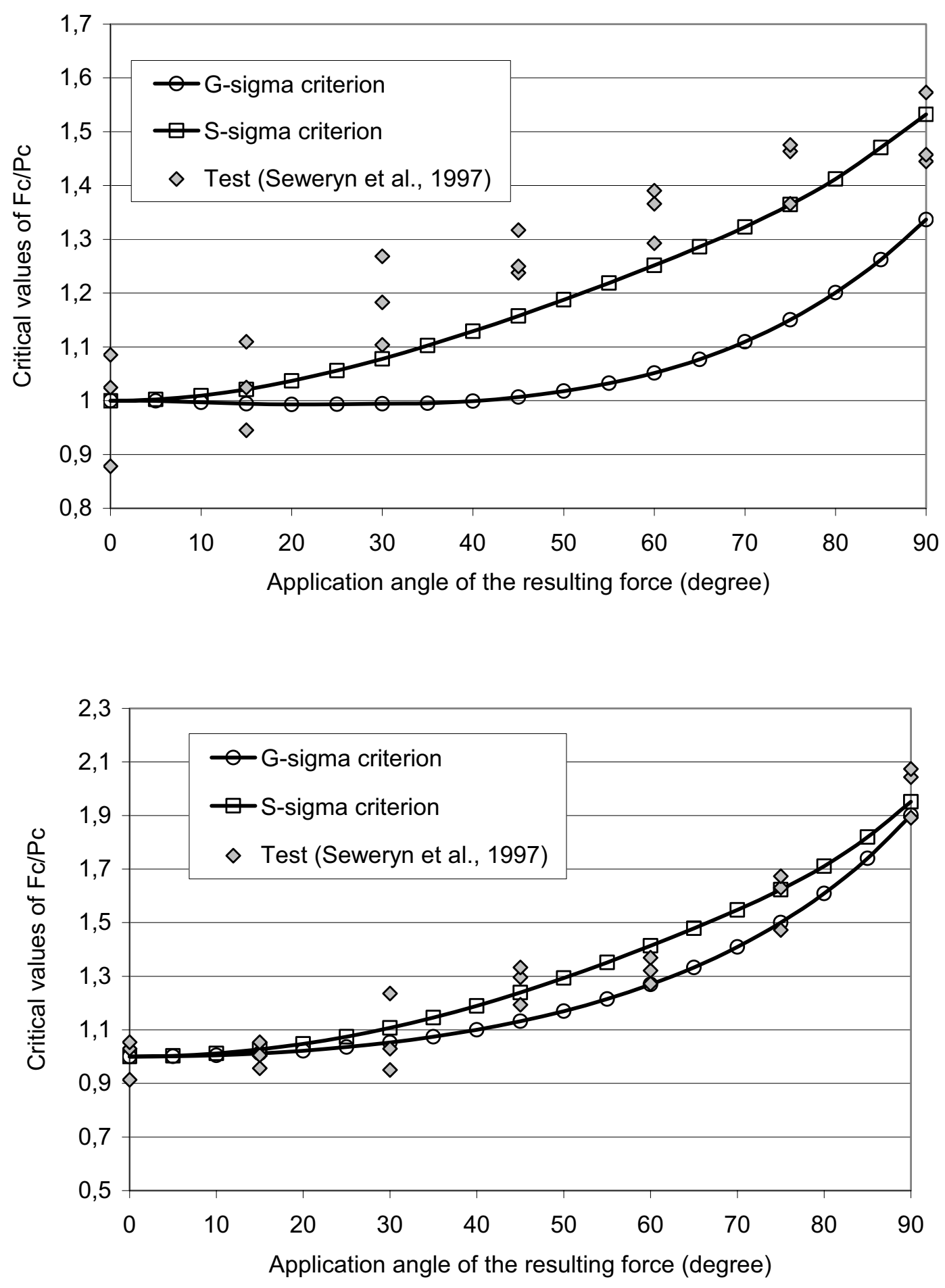

Figure 10. Critical values of $F_{c} / P_{c}$ versus application angle of resulting force: (top) $\beta=10^{\circ}$; (bottom) $\beta=20^{\circ}$. 

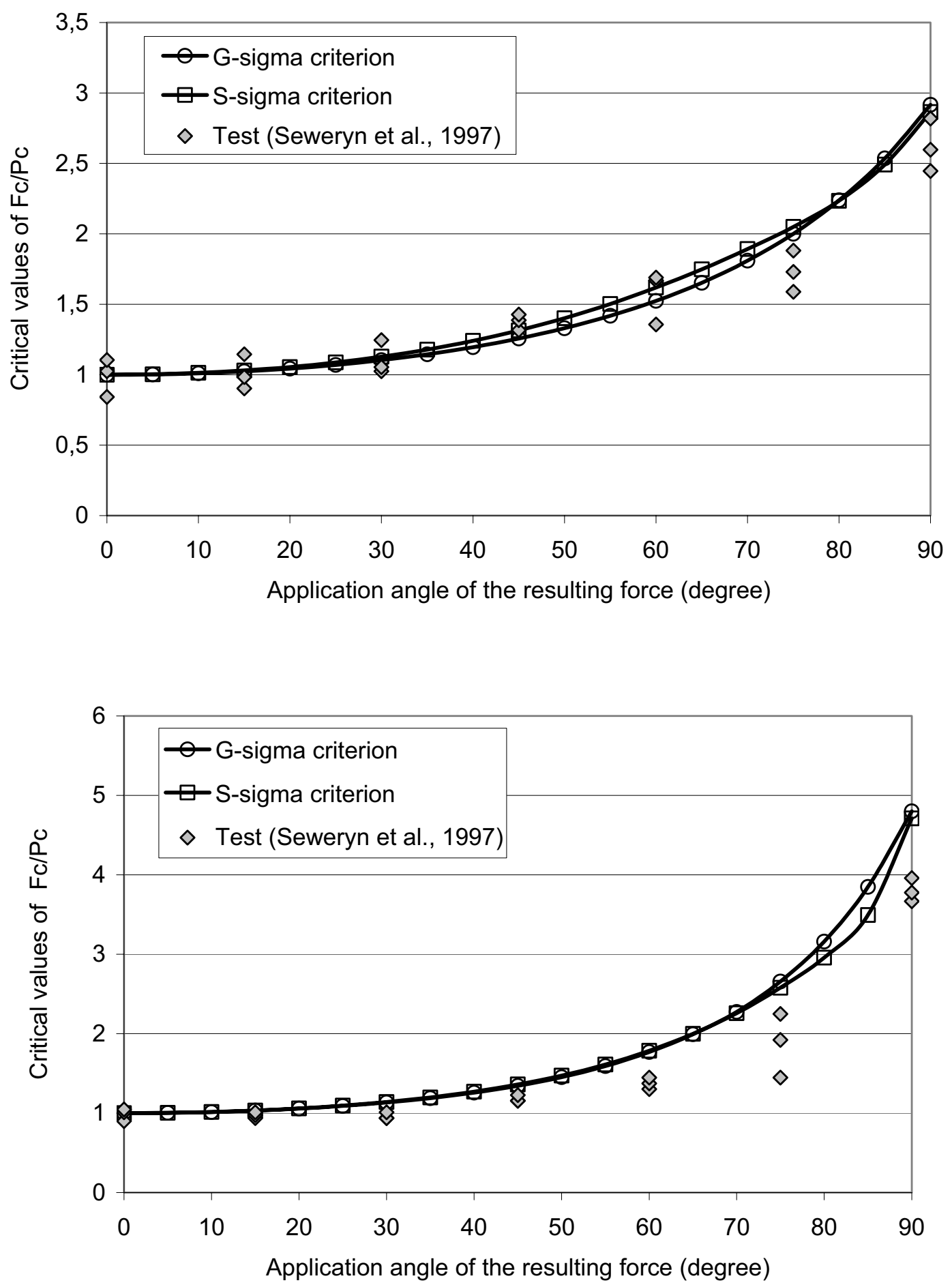

Figure 11. Critical values of $F_{c} / P_{c}$ versus application angle of resulting force: (top) $\beta=30^{\circ}$; (bottom) $\beta=40^{\circ}$. 


\section{Concluding remarks}

In this work, the crack initiation from a notch tip under mixed loads was considered. Two phenomenological criteria were applied to predict the crack initiation. These two criteria, namely the $G-\sigma$ criterion and the $S$ - $\sigma$ criterion, were established by using the concept proposed by [Leguillon 2002], in which both the stress and the energy balances are taken into consideration in the simulation of the crack initiation. The common advantage of the criteria is their self-consistency.

The $G-\sigma$ criterion was established on the analysis of up and low bounds of the critical loading, as a consequence, is physically reasonable. In the present work, by means of an important numerical effort, we have established the indispensable relationships between the strain energy release rate after a crack kinking and the remote loads for a $V$-notch under mixed mode loading. These relationships enable us to apply the $G-\sigma$ in a straightforward manner.

The strain energy density criterion was proposed for crack propagation and bifurcation with successful applications. In the present work, this criterion was modified on the basis of Leguillon's concept to form a criterion capable to predict the crack initiation from a $V$-notch tip. Even though its physical interpretation is less evident, this criterion benefits from its simplicity in applications.

Confrontation with experimental data shows that both the criteria enable reasonably accurate prediction of fracture in elements with $V$-notches subjected to mixed mode loading. Consequently, they can be effectively applied in practical engineering applications.

\section{References}

[Bansal and Kumosa 1998] A. Bansal and M. Kumosa, "Analysis of double edge-cracked Iosipescu specimens under biaxial loads", Eng. Fract. Mech. 59:1 (1998), 89-100.

[Bazant 2000] Z. P. Bazant, "Size effect”, Int. J. Solids Struct. 37:1-2 (2000), 69-80.

[Broughton et al. 1990] W. Broughton, M. Kumosa, and D. Hull, "Analysis of the Iosipescu shear test as applied to unidirectional carbon-fibre reinforced composites”, Compos. Sci. Technol. 38:4 (1990), 299-325.

[Destuynder et al. 1983] P. Destuynder, M. Djaoua, and S. Lescure, "Quelques remarques sur la mécanique de la rupture élastique", J. Mec. Theor. Appl. 2 (1983), 113-135.

[Dunn et al. 1997a] M. Dunn, W. Suwito, and S. Cunningham, "Fracture initiation at sharp notches: correlation using critical stress intensities”, Int. J. Solids Struct. 34:29 (1997), 3873-3883.

[Dunn et al. 1997b] M. Dunn, W. Suwito, S. Cunningham, and C. May, "Fracture initiation at sharp notches under mode I, mode II and mild mixed mode loading”, Int. J. Fract. 84:4 (1997), 367-381.

[Irwin 1968] G. Irwin, "Linear fracture mechanics, fracture transition and fracture control", Eng. Fract. Mech. 1:2 (1968), 241-257.

[Kosai et al. 1993] M. Kosai, A. Kobayashi, and M. Ramulu, Tear straps in airplane fuselage, durability of metal aircraft structures, edited by S. Atluri et al., Atlanta Technology Publishers, 1993.

[Leguillon 2002] D. Leguillon, "Strength or toughness? A criterion for crack onset at a notch", Eur. J. Mech. A Solids 21:1 (2002), 61-72.

[Leguillon and Yosibash 2003] D. Leguillon and Z. Yosibash, "Crack onset at a v-notch. Influence of the notch tip radius", Int. J. Fract. 122:1-2 (2003), 1-21.

[McClintock 1958] F. McClintock, "Ductile fracture instability in shear", J. Appl. Mech. (Trans. ASME) 25 (1958), $582-588$.

[Mroz and Seweryn 1998] Z. Mroz and A. Seweryn, "Non-local failure and damage evolution rule: application to a dilatant crack model", J. Phys. 8 (1998), 257-268.

[Palaniswamy and Knauss 1972] K. Palaniswamy and W. Knauss, "Propagation of a crack under general in-plane tension", Int. J. Fract. 8:1 (1972), 114-117. 
[Reedy and Guess 1995] J. E. Reedy and T. Guess, "Butt tensile joint strength: interface corner stress intensity factor prediction”, J. Adhes. Sci. Technol. 9 (1995), 237-251.

[Ritchie et al. 1973] R. Ritchie, J. Knott, and J. Rice, "On the relationship between critical tensile stress and fracture toughness in mild steel", J. Mech. Phys. Solids 21:6 (1973), 395-410.

[Seweryn and Lukaszewicz 2002] A. Seweryn and A. Lukaszewicz, "Verification of brittle fracture criteria for elements with $V$-shaped notches", Eng. Fract. Mech. 69:13 (2002), 1487-1510.

[Seweryn et al. 1997] A. Seweryn, S. Poskrobko, and Z. Mroz, "Brittle fracture in plane elements with sharp notches under mixed-mode loading", J. Eng. Mech. 123:6 (1997), 535-543.

[Sih 1974] G. Sih, "Strain-energy-density factor applied to mixed mode crack problems", Int. J. Fract. 10:3 (1974), $305-321$.

[Suo and Combescure 1992] X. Suo and A. Combescure, "On the application of $G-\theta$ method and its comparison with De Lorenzi’s approach”, Nucl. Eng. Des. 135:2 (1992), 207-224.

Received 2 Dec 2005.

JIA LI: jia.li@lpmtm.univ-paris13.fr

LPMTM, CNRS UPR 9001, Institut Galilée, Université Paris 13, 99 Avenue Jean-Baptiste Clément, 93430 Villetaneuse, France

XIAO-BING ZHANG: zang@moniut.univ-bpclermont.fr

LAMI, Université Blaise Pascal de Clermont-Ferrand, Avenue Aristide Briand, 03100 Montluçon, France 